\title{
ONE HUNDRED AND FORTY-ONE CASES OF RECUR- RENT VOMITING IN PRIVATE PRACTICE *
}

\author{
CHARLES GILMORE KERLEY, M.D. \\ NEW YORK
}

The cases reported have been seen in private practice during the past eight years. Cases seen in consultation with other physicians have not been included.

The history diagnosis was based on definite periodic attacks of vomiting, for which condition the patient was brought to me.

Recurrent vomiting, in a vast majority of the cases, we found occurs in the offspring of those who for two or more generations have not been occupied with manual work. The recurrent vomiting patients are the offspring of those who have had business or professional occupation.

\section{SUMMARY OF THE FEATURES OF CASES SEEN}

Average time cases were followed was 104 weeks.

Sex: Seventy were boys; seventy-one were girls.

Family History: Rheumatism in one or both parents in forty; sick headache and bilious attacks in forty.

Appetite: In 31 per cent. was normal; in 69 per cent. it was indifferent; in 59.5 per cent. there was constipation.

Previous Feeding in 120 Cases: Twenty-one patients were nursed by mother nine months; forty were nursed by mother six months; four cases had been wet-nursed; sixty-nine had been difficult feeders on cow's milk and had been given the usual trial of foods.

Recurrent colds had been present in 41 per cent. This information was usually volunteered. In twelve there was definite history of eczema; six had habit tic. In fourteen there had been or was enuresis. In thirteen there had been rheumatism.

Urine: Nothing was known as to the condition of the urine in the attacks before coming under observation. The presence of acetone in the later attacks was noted with very few exceptions, but there were exceptions. A child in an attack may show acetone and in the next attack the urine may be free.

Onset of the Vomiting Seizures: Thirty-seven occurred during the first year; twenty-four occurred during the second year; twenty-one occurred during the third year. Onset in the youngest was 6 months. In three the onset was during the eighth year.

Duration of Interval Between Attacks in 119 Cases: Under 2 weeks, 22; from 2 to 4 weeks, 27 ; from 4 to 6 weeks, 10 ; from 6 to 12 weeks, 42 ; from 3 to 6 months, 40 .

Duration and Severity of Seizures: The severity of the attacks varied widely; in some the child would vomit but two or three times. These usually represented the short-interval cases. In others the vomiting was protracted and severe. The average was three and one-half days. The longest seizure under observation continued thirteen days.

* Read at the annual meeting of the American Pediatric Society, Stockbridge, Mass., May 28, 1914. 
The average interval in 118 cases was eight and one-fourth weeks.

Temperature: $102 \mathrm{~F}$. or over in $30 ; 103 \mathrm{~F}$. or over in $10 ; 104 \mathrm{~F}$. or over in $6 ; 105 \mathrm{~F}$. or over in 3 . In the remainder there was an elevation of from 100 to $102 \mathrm{~F}$.

In taking the history in fifteen cases, the mother or attendants volunteered the information that the child had a peculiar breath at the time of the attack which we interpreted as the so-called "acetone breath."

Onset: Average age in 121 cases was 2 years and 8 months; average number of attacks in 99 cases had been 9; average age brought for treatment was 5 years.

Results: Of cases in which there were no attacks after beginning treatment: Forty-one were followed for from two to eight years. Of these fortyone, twenty-six were followed from three to eight years and fifteen were followed from two to three years. Fifteen were followed from one to two years; nine cases were followed from six months to one year; eighteen cases were followed less than six months. Several of the latter are recent cases in which decided gain in weight and general improvement has occurred. In nine cases there was very much improvement; the attacks were mild and comparatively infrequent. Of these eight were followed for from two and one-half to eight years; one was followed eighteen months. In thirty-three cases there was improvement; the attacks less frequent and less severe. Of these thirteen were followed from two and one-half to eight years; six were followed from one year to eighteen months. The remaining fourteen of this group were followed for varying periods under one year, but the records show that a decided impression had been made on the seizures.

In sixteen cases there was no apparent improvement. In some the treatment was indifferently carried out and the patient was dismissed. In others, no apparent impression was made on the disease and the seizures continued as before.

I have an unimproved case that has been under treatment for three years without benefit. The attacks continue to be very severe at from ten to twelve weeks' interval. I only recently was successfull in having massage and exercise treatment instituted.

Another boy now aged 6 has attacks of either periodic fever, bronchitis or recurrent vomiting, rarely going for three months without an explosion of some kind. He has been my patient since he was 6 months of age.

In a vast majority of the cases the recurrence will be completely controlled if continued family cooperation is secured. A diet and living regimen will be followed faithfully by some parents as long as they can be kept anxious about the patient. When the child has passed three or four vomiting periods, the use of forbidden articles of food is often gradually resumed; the former methods of living are more attractive than the regimen laid down.

The association of recurrent vomiting with other forms of illness is interesting. As mentioned before, 41 per cent. of these children were subject to repeated bronchitis, usually of the spasmodic type, and not infrequently the bronchitis and the vomiting occurred simultaneously. Acetone was not present in these cases unless gastric symptoms were present. In others, the attacks will be distinct and separate. In one 
patient there was in nearly every attack an acute spasmodic laryngitis; bronchial asthma very urgent followed and this again was followed sometimes, but not invariably by repeated vomiting. This child had suffered severely with eczema when an infant. Two boys, brothers, were of particular interest in that they had the attacks simultaneously, beginning with tonsillitis, followed by bronchitis and asthma and ending with recurrent vomiting lasting for a day or two. In a few cases the recurrent vomiting attacks were replaced by high fever for three or four days, with acetonuria and without other symptoms.

Two girls, aged 5 years, both in wretched condition, had mild vomiting every week or ten days. In both the acetone breath and acetone urine were continuously present.

The only fatal case seen by me and not included in the above statistics was in a girl 7 years of age living in a New York suburb. There had been previous attacks at about from three to six months' intervals. This attack was particularly severe, no fever but frequent and violent emesis. The vomiting had continued about four days when I saw her. At this time there was considerable exhaustion, but the child was fairly bright, answered questions and talked freely. I endorsed the attending physician's treatment, made a favorable prognosis, and the child died in five hours. Apparently, death was due to respiratory paralysis.

Three patients had been operated on for appendicitis in Paris without relief. A normal appendix was removed in each case. In three it was necessary to resort to morphin hypodermatically to control the vomiting seizures.

MANAGEMENT DURING THE INTERVAL

The management in the main was the same in all, and one reason for reporting only the cases seen during the past eight years is that during this period practically the same interval management has been carried out.

Diet: If the case is a pronounced one, the patient is given a diet with few restrictions, except that cow's milk, butter, cream and sugar are omitted. One egg is allowed perhaps every third day. Saccharin is permitted as a sweetening agent in some and very little sugar in any case.

Three meals daily are allowed with nothing between meals. Red meat is given scantily three times a week. Poultry and fish are given at other times. In some skimmed milk is allowed scantily, never more than one pint daily, often less. Puddings are made with skimmed milk. A grave error in our management of many children is the free use of cow's milk, butter, ice-cream and sugar. I could present dozens of records showing surprising gain in weight and marked improve- 
ment in the general well-being of the patient after a considerable withdrawal of milk, cream and sugar from the diet.

The period of lactation in the human being is at the most a year and then the child is ready for other food than milk. I believe that the average well child would thrive far better if he were to get not more than one pint of milk daily after the fifteenth month. Sugar was not used except as a condiment as we now use honey, until three hundred years ago. Unknown millions lived their span without it.

Medication: The further treatment consists in the internal use of salicylate of soda, bicarbonate of soda, independent or in combination as advocated by Rachford. In a pronounced case I give 5 grains of sodium salicylate with 10 grains of sodium bicarbonate three times daily at five-day intervals, or 20 to 30 grains of the sodium bicarbonate daily for a month or two at first. This drug treatment is carried on with rest periods for months and years as the case may require.

Bodily Exercise: A very important factor in the management is in arranging daily physical exercise, such as riding horseback or the bicycle and walking so many street blocks a day. A warm bath and a brisk rub are given at night, and last but not the least important feature in the treatment is the use of physical therapeutics. In severe cases I use if possible daily massage together with various body manipulations and exercises, the latter sufficient to make the child perspire, but not to the point of exhaustion. The advantage of this phase of the treatment was called to my attention by Dr. W. P. Northrup.

Bowel Function: A daily evacuation of the bowels is insured by suitable measures.

Some children I take out of school. For others I advise a modified rest-cure, which means in bed until 10 a. m., rest one and one-half hours after dinner and in bed and lights out at 6 or $7 \mathrm{p} . \mathrm{m}$. The nervous element in these cases is not to be forgotten. If the attendant is not agreeable to the patient her services are dispensed with. In some a temporary elimination of the mother has been of assistance. The precipitation of an attack by fatigue and fright is not unusual.

The regulation of the life and habits aids materially in the management, but is of little or no use if the carbon content in the food is not reduced to the oxidizing possibilities.

\section{MANAGEMENT DURING THE ACUTE VOMITING ATTACK}

$I$ find that in the vast majority of the cases, a weak solution of sodium bicarbonate is best retained in the strength of 5 grains of the bicarbonate to 8 ounces hot water. This is given freely.

As laxatives the magnesia preparations are best retained and are used when a laxative is required. Calomel or mercury with chalk will 
often increase the vomiting. In other cases their use supplies very material assistance. When the vomiting has continued for twenty-four to thirty-six hours the patient is given colonic flushings with sodium bicarbonate, 2 drams to 8 ounces of water at eight-hour intervals. The solution is best retained if it is given warm (105 F.), the tube inserted from 8 to 12 inches.

Feeding: Nothing is gained by attempts at forcing the feeding. When the child is ready for food, he is given barley or rice gruel with dried bread crusts or unsweetened zweiback.

Judging from the results obtained through the withdrawal of highly energized foods and in the use of active and passive exercises it would seem that the chief error in most cases rests in a defective oxidation, or in the giving of food substances of high carbon content in excess beyond the powers of normal oxidation.

In this connection the views of Francis $\mathrm{Hare}^{1}$ are particularly interesting. Hare states that carbon intake must be offset by carbon expenditure or energy intake with compensating energy expenditure, if "hyperpyremia" (excess of fuel in the blood) is not to occur. When physiologic functions are deficient in maintaining a balance, and an excess of carbon or hydrocarbon is not wholly applied in the manufacture of additional fat, bile, milk or other secretion, or lost by exercise or menstruation, a pathologic function is then necessary to free the system of its excess of fuel. As such so-called pathologic functions, Hare mentions gout, migraine, gastralgia, bilious attacks, epilepsy and asthma, all of which operate through either a reduction of income or an increase of expenditure by vomiting, muscular action or fever, periodic in character. These attacks of migraine have been observed alternating in the same individual with attacks of gout; gout has subsided with the development of glycosuria, and women (according to Garrod) show greatest liability to the development of gout shortly after the menopause, one decarbonizing process replacing another.

I am not convinced of the reliability of this theory in its entirety, but find it peculiarly in harmony with the clinical facts under presentation, and (while not forgetting for a moment that proteid anaphylaxis (Meltzer) and reflex neuroses from various abnormalities are still to be considered as operative causes in such affections as asthma, recurrent vomiting and migraine) I wish to emphasize the following suggestive points :

Eczema, spasmodic laryngitis, cyclic vomiting and recurrent bronchitis and asthma are all notoriously frequent in children of gouty or rheumatic ancestry.

1. Hare, Francis: Carbon Factor in Gout, Med. Rec., June 17, 1905. 
All these conditions are met less frequently and with diminishing severity at the age of puberty when the processes of combustion and tissue building are at their maximum.

While two or more of these so-called pathologic functions are not ordinarily observed simultaneously in the same child, not a few children suffer from a number of these conditions in alternation over a period of years. In other words, the processes seem to be mutually compensatory.

In the winter months, when activity is lessened and perspiration is least, every one of the conditions mentioned is intensified.

Finally, in every instance, diet (as the cases show) is a most prominent factor influencing the susceptibility of the patient.

The statistics as presented were prepared from my case histories by my associate, Dr. Frank Elmer Johnson, to whom I wish to express my indebtedness.

132 West Eighty-First Street. 